\title{
A case of obstructive sleep apnea syndrome associated with floppy eyelid syndrome: positive effect of CPAP therapy
}

\author{
Roberto Porta1, Laura Comini2, Luca Barbano', Luca Bianchi1, Michele Vitacca1 \\ ${ }^{1}$ Respiratory Rehabilitation Division, Maugeri Clinical Scientific Institutes, IRCCS Lumezzane, Lumezzane (BS) \\ 2 Health Directorate, Maugeri Clinical Scientific Institutes, IRCCS Lumezzane, Lumezzane (BS), Italy
}

\begin{abstract}
The obstructive sleep apnea syndrome (OSAS) may be associated with several eyes disorders, among which the most common is the floppy eyelid syndrome (FES). We intended to highlight the association between OSAS and FES and evaluate the effect of FES treatment with Continuous Positive Airway Pressure (CPAP).

A 50-year patient with a 10-year history of snoring, sleep fragmentation and daytime sleepiness associated with several comorbidities has been studied. For six months, several ocular symptoms were present, particularly on waking up in the morning. An overnight respiratory polygraphy was performed at baseline and after CPAP titration.

The treatment with CPAP corrects apnea/hypopnea events and rapidly improves patient's daytime sleepiness and eyes FES-related symptoms. This improvement is already evident after a very short period of treatment.
\end{abstract}

\section{Introduction}

The Obstructive Sleep Apnea Syndrome (OSAS) is the more common form of sleep-disorders breathing characterized by repetitive partial or complete closure of the upper airway during sleep, including arterial

Corresponding author: Dr. Roberto Porta, Respiratory Rehabilitation Division, Maugeri Clinical Scientific Institutes, Via G. Mazzini 129, 25065 Lumezzane (BS), Italy. Tel. +39.030 .8253169 - Fax +39.030 .8253189 .

E-mail: roberto.porta@icsmaugeri.it

Key words: Obstructive sleep apnea; floppy eyelid syndrome; ocular diseases; palpebral laxity; continuous positive airway pressure.

Contributions: RP, conception and design, data analysis; RP, LC, LBa, LBi, MV data interpretation, article drafting or critical revision for important intellectual content. All authors approved the final version to be published.

Conflict of interest: the Authors declare no conflict of interest.

Received for publication: 23 August 2016

Accepted for publication: 11 December 2016

(C) Copyright R. Porta et al., 2017

Tipografia PI-ME Editrice, Italy

Monaldi Archives for Chest Disease 2017; 87:766

doi: 10.4081/monaldi.2017.766

This article is distributed under the terms of the Creative Commons Attribution Noncommercial License (by-nc 4.0) which permits any noncommercial use, distribution, and reproduction in any medium, provided the original author(s) and source are credited. oxygen desaturation, sleep fragmentation and daytime sleepiness [1]. Sleep disorders can be associated with eyes disorders as glaucoma, optic neuropathy, papilledema, corneal abnormalities and non-arteritic anterior ischemic optic neuropathy [2].

Kadyan et al. [3] in their study observed that eye rubbing, gritty sensation, mucoid discharge, and photophobia were significantly more frequent in the OSAS patients in comparison to the control group. In this study, only four patients with OSAS (4.5\%), but none of the control group, experienced spontaneous lid eversion when sleeping, squeezing, or rubbing eyes. More recently, other Authors reported floppy eyelid syndrome (FES) [4] as one of the more common eye diseases associated with OSAS. FES was firstly described in 1981 by two ophthalmologists, Culbertson and 0stler [5], as an unusual entity characterized by 'floppy' and redundant upper eyelid with marked papillary conjunctivitis in obese middle-aged and older men. FES is an under-diagnosed disorder of unknown pathogenesis, whose prevalence in patients with OSAS is not negligible.

Chambe et al. [4], in a prospective study on 127 patients, observed FES in 22.8\% of their OSAS population, being FES prevalence higher in severe OSAS [4.] Controversial findings are reported about the relationship between FES and OSAS. However, why patients with OSAS are at risk for FES is not known [6].

The association between FES and OSAS has both diagnostic and therapeutic implications. The current case-study shows how an appropriate OSAS treatment with Continuous Positive Airway Pressure (CPAP) did not only result in correction of apnea/hypopnea and improvement in night-time and day-time symptoms, but also brought a quick benefit of FES-related symptoms.

\section{Case Report}

A 50-year-old man suffering from hypertension, hypercholesterolemia and mild obesity (BMI $32.7 \mathrm{Kg} \mathrm{m}^{-2}$ ), with a 10-year history of snoring, sleep fragmentation and daytime sleepiness was admitted as outpatient in a sleep-dedicated ambulatory in our Respiratory Rehabilitation Division. In the last three years, his symptoms had been worsened with the appearance of nocturia, waking gasping for air during night and headache in the morning. The diurnal symptoms were daytime sleepiness, with Epworth Sleepiness Scale (ESS) equal to 14/24, and asthenia - with a consequent difficulty in concentration - that had caused loss of job performance (the patient was a personal computer worker). The patient had also complied ocular symptoms as redness, burning eyes and nonspecific irritative ocular symptoms arising on waking up (Figure 1). In the six months before the visit, xerophthalmia in presence of burning and pain, puffy eyes, amblyopia and convergent strabismus upon awakening occurred. Sjogren's syndrome and autoimmune diseases were excluded. The symptoms regressed with the use of proper eye drops, but did not resolve despite different ophthalmological examinations. Only an ophthalmologist, suspecting nocturnal hypoxia, suggested to perform polysomnography. 


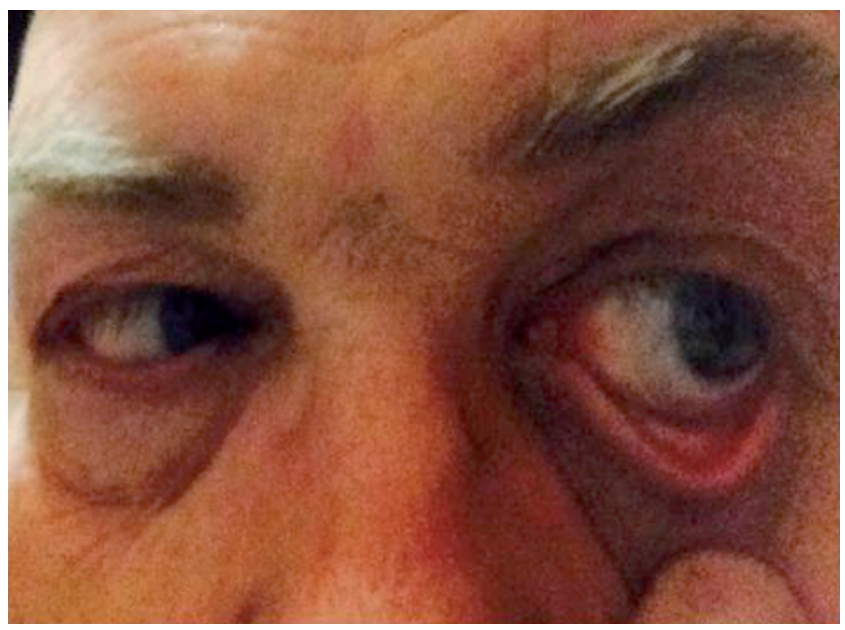

Figure 1. Evidence of FES in the unsuspected OSAS patient.

The pulmonologist performed chest examination and found the range of Mallampati score to be normal. The ocular signs (i.e., intense conjunctival hyperemia and palpebral laxity) were confirmed. Blood emogas-analysis, performed in sitting position, showed $\mathrm{pH} 7.42, \mathrm{PaO}_{2}$ $80 \mathrm{mmHg}$ and $\mathrm{PaCO}_{2} 41 \mathrm{mmHg}$. An overnight respiratory portable polygraphy was provided to the patient and, the day after, the recordings were scored manually showing a severe OSAS, with an apnea/hypopnea index (AHIa) of 90.3/hour. Nocturnal oximetry showed a severe impairment in saturation data: $\mathrm{mSaO}_{2} 86 \%$, min $\mathrm{SaO}_{2} 75 \%$ and $\mathrm{CT}_{90}$ $63 \%$. The patient was admitted to hospital and began the CPAP titration using an auto-titrating positive airway pressure (autoCPAP) device (REM Star Respironics). After only four nights, the ocular problems were completely disappeared and a fixed CPAP value of $11 \mathrm{cmH}_{2} \mathrm{O}$ was set for a couple of nights. The treatment efficacy was confirmed by a further overnight respiratory assessment showing a significant reduction in AHIa to 6.8/hour (-75\% vs baseline index), and a central apnea index of $2.9 /$ hour. Nocturnal oximetry data significantly improved: $\mathrm{mSaO}_{2} 94.2 \%$, min $\mathrm{SaO}_{2} 88 \%$ and $\mathrm{CT}_{90} 4 \%$. Daytime sleepiness, asthenia and ESS (from 14/24 to 2/24) were also greatly reduced.

Surprisingly, the patient's subjective improvement was very rapid. Indeed, we did not expect that only four nights of autoCPAP could totally delay the irritative and burning eyes symptoms, eye pain and morning amblyopia. The fixed-CPAP setting definitely solved the respiratory pattern and was prescribed as therapy at home. After four months, an ambulatory follow-up visit confirmed the correct adherence of CPAP therapy and that absence of eyes symptoms has been maintained overtime.

\section{Discussion}

Some investigations $[4,7]$, show that severe OSAS is common in patients with FES and significantly relates with, suggesting OSAS might be an independent risk factor both for FES and eyelid hyperlaxity. Wang et al. [6] in a recent meta-analysis confirmed that FES is very common in OSAS, but it is not within the general non-OSAS population; FES risk is 4.12 times higher in OSAS than in non-OSAS individuals.

Pathogenic mechanism of eyelid laxity has not yet been clarified. There is evidence of a substantial loss of elastic fibers and ultrastructural anomalies in the residual fibers of the tarsal plates of patients with FES [8]. On the other hand, there is a correlation between the rate of OSAS and the disorganization of the elastic fiber reticule of the distal uvula tissue [8]. These histopathologic alterations and the rela- tionship between FES and OSAS seem to support the hypothesis that both processes could be different manifestation of the same condition.

Based on the speculative etiology of FES many different treatments have been considered. The usual goal of FES treatment is ophthalmological with topic frequent instillation of artificial tears and ocular lubricants. Only in case of significant cornea or conjunctival damage, the use of local antibiotic is preferred. Until now, there are few studies suggesting the effect of CPAP treatment on reversal of FES-related symptoms on the eye and ocular surface [3,9].

Acar et al. [10] performed CPAP treatment on 51 patients suffering from moderate and severe OSAS for 18 months and the eye examination findings pre- and post-CPAP were compared. In this study the Authors concluded that 'an appropriate CPAP therapy helps to relieve both the systemic findings and the ocular surface problems probably by reducing intermittent hypoxemias and arousals, and providing a better quality of sleep pattern'. However, from this study [10] a long-term CPAP therapy (at least 1 year) is expected to be used for improving the clinical picture of FES and overcoming the problem of ocular surface irritation encountered in the early stage.

In our patient, the efficacy of CPAP therapy has brought to correction of apnea/hypopnea, oxygen nocturnal desaturation and improvement in quality of sleep pattern and day-time symptoms. The current report highlights the rapid and positive effect of CPAP treatment on signs and symptoms of FES associated to OSAS offering an interesting proof for a quick reversal of FES-related symptoms.

Sensibilization on ocular findings in unsuspected patients should be already started by the general practitioner in the community. This would allow the identification of hidden sleeping diseases needing for a more appropriate investigation and possible treatment. Anyway, pulmonologist and ophthalmologist should pay greater attention to patient's ocular symptoms and consider a deeper mutual collaboration.

\section{References}

1. The report of America Academy of Sleep Medicine Task Force. Sleep-related breathing disorders in adults: recommendations for syndrome definition and measurement techniques in clinical research. Sleep 1999;22:667-89.

2. McNab AA. The eye and sleep apnea. Sleep Med Rev 2007;11:269-76.

3. Kadyan A, Asghar J, Dowson L, Sandramouli S. Ocular findings in sleep apnoea patients using continuous positive airway pressure" Eye (Lond) 2010;24:843-50.

4. Chambe J, Laib S, Hubbard J, et al. Floppy eyelid syndrome is associated with obstructive sleep apnoea: a prospective study on $127 \mathrm{pa}-$ tients. J. Sleep Res 2012;21:308-15.

5. Culbertson WW, Ostler HB. The floppy eyelid syndrome. Am J Ophthalmol 1981;92:568-75.

6. Wang P, Yu DJ, Feng G, et al. Is floppy eyelid syndrome more prevalent in obstructive sleep apnea syndrome patients? J Ophthalmol 2016;2016:6980281.

7. Muniesa MJ, Huerva V, Sánchez-de-la-Torre M, et al. The relationship between floppy eyelid syndrome and obstructive sleep apnoea. Br J Ophthalmol 2013;97:1387-90.

8. Schlotzer-Schrehardt U, Stojkovic M, Hofmann-Rummelt C, et al. The pathogenesis of floppy eyelid syndrome: involvement of matrix metalloproteinases in elastic fiber degradation. Opthalmology 2005;112:694-704.

9. McNab AA. Reversal of floppy lid syndrome with treatment of obstructive sleep apnoea. Clin Experiment Ophthalmol 2000;28:125-6.

10. Acar M, Firat H, Yuceege M, Ardic S. Long-term effects of PAP on ocular surface in obstructive sleep apnea syndrome. Can J Ophthalmol 2014;49:217-21. 